\title{
Observations of power line harmonic radiation by the low-altitude AUREOL 3 satellite
}

\author{
Michel Parrot \\ Laboratoire de Physique et Chimie de l'Environnement, Centre National de la Recherche Scientifique, \\ Orléans, France
}

\begin{abstract}
During the past decade, most scientific satellites have been dedicated to the study of the auroral zones, but low-altitude satellites also allow the study of the anthropogenic effects in the mid-latitude regions. AUREOL 3 was such a low-altitude polar-orbiting satellite, with an apogee and perigee of 2000 and $400 \mathrm{~km}$, respectively, which observed power line harmonic radiation (PLHR). The wave experiment named ARCAD 3 provided the waveforms of five components of the electromagnetic field (three magnetic and two electric) in a frequency range between 0 and $1.5 \mathrm{kHz}$. The telemetry was recorded at different stations. During the lifetime of the mission, 940 records, each lasting approximately $12 \mathrm{~min}$, were processed in order to obtain spectrograms of the waveforms. On these spectrograms, five events with magnetospheric lines have been detected, which in all cases are separated by $50 \mathrm{~Hz}$ (with an accuracy of $1 \%$ ); such lines are not at exact harmonics of the $50-\mathrm{Hz}$ power system, but drift in frequency with time. The drift, which is between 1 and $8 \mathrm{~Hz} / \mathrm{s}$, is not constant during an event. A common property of these five events is that they were recorded during periods of very low magnetic activity and were observed mainly in the night sector. Comparison of the characteristics of these PLHR with existing theories concerning the general problem of VLF triggered emissions and sideband stability of whistler mode waves, shows that the drift in frequency is not due to a Doppler effect, but most probably to a non linear interaction between electrons and coherent waves, occurring in the topside ionosphere.
\end{abstract}

\section{Introduction}

Power line harmonic radiation (PLHR) is the ELF and VLF waves radiated by electric power systems at the harmonic frequencies of 50 or $60 \mathrm{~Hz}$. These electromagnetic waves are supposed to amplify and trigger many emissions, and thus to participate in the precipitation of electrons from the slot region $(2<L<3)$ between the inner and outer radiation belts. The PLHR phenomenon has commonly been observed on ground, but observations from satellites are rare.

On the ground, the propagation of PLHR through the magnetosphere was first observed by Helliwell et al. [1975], between an industrialized region, Roberval in Quebec, and its conjugate point, the Siple station in Antarctica. Similar observations were made from different places by Matthews and Yearby [1981] and Kimura et al. [1987]. The radiation consists of several line emissions that do not always appear at exact harmonics of 50 or $60 \mathrm{~Hz}$; on the contrary, they are not spaced at exactly the power system frequency. In some cases, the lines shift in frequency. A peak in PLHR events is observed around $3 \mathrm{kHz}$, and between 0600 and 1800 local time [Park and Helliwell, 1978]. During an experiment with a transmitter, it was found by Park and Chang [1978] that power line effects can be simulated by radiating as little as $0.5 \mathrm{~W}$ at a given frequency. An estimation of the PLHR power radiated into the magnetosphere was measured by Yearby et al. [1983].

Copyright 1994 by the American Geophysical Union.

Paper number 93JA02544. 0148-0227/94/93JA-02544\$05.00
A low-altitude survey of VLF emissions obtained by the Ariel 3 and 4 satellites [Bullough et al., 1976; Tatnall et al., 1983; Bullough et al., 1985] revealed a maximum in wave activity over North America that the authors attributed in part to the PLHR. But the data from these two satellites only consisted of averaged values in three frequency bands $(750 \mathrm{~Hz}, 3.2$ and $9.6 \mathrm{kHz})$, and did not allow direct observations of PLHR. Weak emissions related to PLHR were observed on board the ISEE 1 satellite by Bell et al. [1982] above North America. The line radiation was only detected on 5 of the 90 orbits studied, and were observed during periods of low magnetic activity. Most of these magnetospheric lines were located between 3 and $4 \mathrm{kHz}$, and their frequencies significantly deviated from the PLHR frequencies as was already observed from ground data. During disturbed propagation conditions, one event related to the PLHR was detected on the S3-3 satellite by Koons et al. [1978]. Some observations with the ISIS 2 satellite are given by Park and Helliwell [1981]. In this last case, the line frequencies are less than $1.8 \mathrm{kHz}$. Field measurements of PLHR at the fundamental frequency were made in the atmosphere with balloons [Tomizawa and Yoshino, 1984]. The same experiment in the topside ionosphere, showing increases of the magnetic field strength at the fundamental frequencies 50 and/or $60 \mathrm{~Hz}$, was reported by Tomizawa and Yoshino [1985]. Concerning the AUREOL 3 data, an increase of the VLF activity has also been observed over North America [Parrot, 1990]. It was shown by Parrot et al. [1991] that the intensity of the electric component field that is parallel to the Earth's magnetic field varies with the days of the week, with a maximum on Monday. A theoretical model which ascribes this observation to a PLHR effect was presented by Molchanov et al. [1991]. 
In the observations mentioned above, the problem of frequency shift was attributed either to a Doppler effect or to a non linear wave-particle interaction. This phenomenon was considered in the past by many authors, because it is related to the general problem of VLF-triggered emissions and the non linear interaction between energetic electrons and coherent VLF waves [Helliwell, 1967; Nunn, 1971, 1984; Brinca, 1972a]. At higher frequencies than PLHR, other coherent waves such as the pulses of VLF ground-based transmitters are known to produce similar phenomena [Bell, 1985]. The other important feature with the VLF transmitter signals is that sidebands of electrostatic nature are generated [Nunn, 1973; Tanaka et al., 1987; Bell and Ngo, 1988, 1990]. To explain these observations, Nunn [1986] has presented a nonlinear theory of sideband stability for different ducted whistler mode waves including the PLHR. It is shown that a maximum upward drift of $30 \mathrm{~Hz} / \mathrm{min}$ could be expected if the wave amplitude is larger than $0.5 \mathrm{pT}$. With another simulation, Carlson et al. [1990] found a $30 \mathrm{~Hz} / \mathrm{s}$ frequency drift rate during whistler mode amplification of a wave pulse. A recent review of theoretical and numerical studies of VLF-triggered emissions, generated by man-made signals and propagating in the whistler mode in the magnetosphere, is given by Omura et al. [1991]. Helliwell [1988] has reviewed the main characteristics of the controlled VLF signals injected into the magnetosphere from Siple and received on ground or satellites. A survey of the impact of PLHR on natural noise in our environment is given by Kikuchi [1983]. It was shown by $S a ̀$ [1990] that when a wave is perturbed by a weaker signal (PLHR), sidebands, and consequently triggered emissions can occur even if the amplitude ratio between the two signals is as high as $50 \mathrm{~dB}$.

This paper studies magnetospheric lines observed by the lowaltitude orbiting AUREOL 3 satellite. It is shown that these lines are related to PLHR. The wave experiment on AUREOL 3 and the observed events are described in section 2 . Section 3 presents the comparison with past observations made on the ground as well as from satellites. The existing theories that can explain the main characteristics of the AUREOL 3 observations are discussed in section 4 . Conclusions are presented in section 5 .

\section{The Data}

The wave experiment ARCAD 3 was placed into a quasi-polar orbit on board the triaxial-stabilized AUREOL 3 satellite (apogee $2012 \mathrm{~km}$, perigee $408 \mathrm{~km}$, inclination 82 ${ }^{\circ} 5^{\prime}$, period $109.5 \mathrm{~min}$ ), launched on September 21, 1981. ARCAD 3 measured three magnetic and two electric components of the waves in the ELF and VLF range, from $10 \mathrm{~Hz}$ up to $15 \mathrm{kHz}$. The $E_{z}$ component was approximately parallel to the Earth's magnetic field at high latitude, the $E_{H}$ component being nearly perpendicular. The $x$ direction was nearly parallel to the velocity of the satellite. All characteristics of the experiment are given by Berthelier et al. [1982]. Two telemetry systems were available on AUREOL 3. One was the real-time French telemetry (TMF), only used when the satellite was near a telemetry station, the second was the on board tape-recorded Soviet telemetry (TMS). In contrast to the TMS, the TMF contained high-bit rate data and provided waveforms of five components of the electromagnetic field in a $10 \mathrm{~Hz}-1.5 \mathrm{kHz}$ bandwidth. The sampling frequency $f_{s}$ was 5000 $\mathrm{Hz}$, which means that the maximum frequency of the spectrum theoretically was $2500 \mathrm{~Hz}$ if we ignore the antialiasing filter.

For this paper the study only concerned the TMF data recorded at different telemetry stations. Data from October 1981 until
December 1983 were used. Considering all telemetry stations, there are approximately 940 TMF records each lasting between 12 and $15 \mathrm{~min}$. The recording periods were not selected and varied from one day to another, depending on the programmation of the experiment and on the position of the stations. Using all recorded data, spectrograms were made on a routine basis with a frequency resolution of $2.5 \mathrm{~Hz}$ and a time resolution of $80 \mathrm{~ms}$. A search for the presence of spectral lines was carried out by visual inspection of these spectrograms of the five available components.

A first example is given in Figure 1. This spectrogram represents the spectrum intensity of the electric signal $E_{\mathrm{Z}}$ received at the Toulouse station on April 9, 1982, as a function of frequency and time. Main geophysical parameters are displayed below. A set of lines drifting upwards is observed between 400 $\mathrm{Hz}$ and $1 \mathrm{kHz}$. A spurious line due to an interference on board the satellite can be seen around $160 \mathrm{~Hz}$. This interference cannot be blended with magnetospheric lines as the frequency was constant during the recording. Another example of magnetospheric lines recorded on December 23, 1981 is given in the presentation of the first results of the ARCAD 3 experiment (Figure 19 of Berthelier et al., [1982]).

Among the 940 considered records, only five events were detected. The characteristics and locations of these observations are summarized in Table 1 . For each event, the following parameters are shown: day, hour (UT) of the beginning of the observation, geographic latitude and longitude, altitude of the satellite, $\mathrm{L}$ value, magnetic local time, $\Sigma K p$ or the sum of the $K p$ indices for the day, a $K p$ index corresponding to a period of 3 hours, and the maximum number of observed lines. The number of lines was detected by spectral analysis of the waveform data. An example of the spectrum obtained on December 23 at 0402:31 UT is plotted in Figure 2. The frequency range is between 700 and $1300 \mathrm{~Hz}$, and the frequency resolution of analysis is $2.44 \mathrm{~Hz}$. All peaks numbered from 1 to 9 are roughly distant by $50 \mathrm{~Hz}$. It must be noted that the peaks have different amplitudes.

The recorded signal is eminently nonstationary; to improve the frequency resolution, the accuracy of the spectral analysis was increased using the following DFT (discrete Fourier transform):

$$
X(f)=\sum_{n=0}^{N-1} x(n) e^{-2 x f m \Delta}
$$

where $x(n)$ are the samples, $f$ is the frequency, $\Delta t$ is the sampling interval (1/5000), and $N$ is the number of samples taken into account. Thus the spectrum $S(D)$ is obtained using the equation (1) by

$$
s(f)=X(f) X^{*}(f)
$$

where the asterisk denotes the complex conjugate. A frequency resolution of $0.1 \mathrm{~Hz}$, and a time resolution of $0.5 \mathrm{~s}$ was chosen for each spectrum. To save computer time, the spectra have only been calculated in the frequency band where the lines are observed. In these spectra, it consistently appears that the spectral lines are distant by $50 \mathrm{~Hz}$ with an accuracy of $1 \%$. For two events, the frequency of the peaks has been plotted against time in Figures 3 and 4 . The entire observation period was considered. For a given event, the time of observation of these lines is relatively important (5 min on December 23, 6 min on April 9, 7 min on September 30). It was only limited by the duration of the record or the frequency range of the spectrograms, as the 


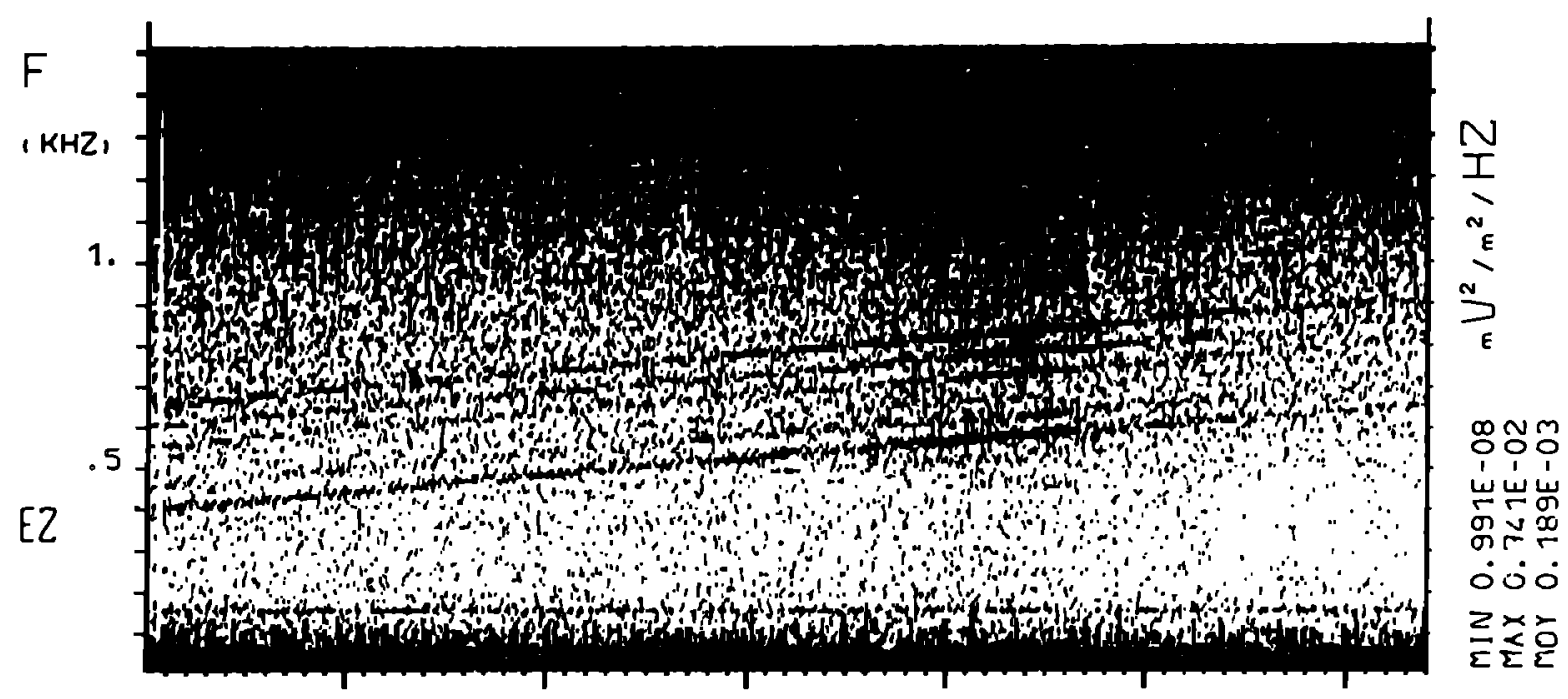

$\begin{array}{llll}\text { UT } & 02.11 .44 & 02.12 .14 & 02.12 .44 \\ \text { MLT } & 4.34 & 4.33 & 4.32 \\ \text { ALTITUDE } & 1789.7 & 1802.6 & 1815.0 \\ \text { INV. LAT } & 43.56 & 42.49 & 41.47 \\ \text { GEOG. LAT } & 39.59 & 38.20 & 36.82 \\ \text { GEOG. LONG } & 23.17 & 23.35 & 23.51 \\ \text { L } & 1.90 & 1.84 & 1.78\end{array}$

Figure 1. Spectrogram of the signal of an electric component recorded on April 9, 1982, during 1 min. Minimum, maximum, and average amplitudes are given on the right. The following parameters are displayed on the bottom: UT time, magnetic local time, altitude in kilometers, invariant latitude, geographic latitude and longitude, $L$ value. PLHR with drifting frequencies are observed between 400 and $900 \mathrm{~Hz}$.

lines drifted upwards or downwards. The regular blanks observed in Figures 3 and 4 are due to the on-board calibration which was removed from the data. The solid line at the bottom of the spectrograms represents the variation of the proton gyrofrequency $f_{H}$ given by a model. Figure 3 concerns the event recorded on April 9, which was partially presented in Figure 1, and where the lines drift upwards. It must be noted that during this event, two different sets of frequencies were observed. At 0211:40 UT, the exact frequencies were $322.3,372.6$, and $422.9 \mathrm{~Hz}$, as well as $529.5,579.4$, and $629.4 \mathrm{~Hz}$. Figure 4 represents a case where the lines drift downwards. It concerns the event recorded on December 23, which was partially shown in Figure 19 of Berthelier et al. [1982]. Up to nine lines are observed, and, for example, the frequency values of the peaks observed at 0358:21 UT are 1368.6, 1418.7, 1468.7, 1518.0, 1568.8, 1618.6, 1668.4, 1718.2 , and $1768.4 \mathrm{~Hz}$. The frequency variations are not so smooth as in the first event, and a large jump is observed at 0400:39 UT.
In the spectrograms of both events, the amplitude of the peaks seems to vanish at low frequencies, and a careful check of the amplitude was made. For each of the two events an average spectrum was obtained for the entire observation period. The amplitudes of the peaks, which are shown in Figures 3 and 4, have also been averaged in frequency taking into account frequency bands of $100 \mathrm{~Hz}$. The two spectra have been plotted in Figure 5 with a dashed curve for the April 9 event, and a dotted curve for the December 23 event. The shape of these spectra seems unusual as the amplitude increases at high frequencies. Considering that the attenuation of the signal is only $-30 \mathrm{~dB}$ at $3000 \mathrm{~Hz}$, attention was paid to the slope of the antialiasing filter. Therefore the full lines in Figure 5 represent for each case what would be the attenuation of the maximum value of both spectra, as a function of the frequency $f$, if we consider that this value is observed at the frequency $5000 \mathrm{~Hz}-f$. There is a quite good agreement between the curves deduced from the slope of the filter and the experimental curves. This indicates that, because of an

TABLE 1. Date, Location, and Main Geophysical Parameters of the Five Events

\begin{tabular}{|c|c|c|c|c|c|c|c|c|c|}
\hline Date & $\begin{array}{c}\text { Hour, } \\
\text { UT }\end{array}$ & Latitude & Longitude & $\begin{array}{c}\text { Altitude, } \\
\text { km }\end{array}$ & $L$ & MLT & $\Sigma K p$ & $K p$ & $\begin{array}{l}\text { Number } \\
\text { of Lines }\end{array}$ \\
\hline Nov. 5, 1981 & $2247: 43$ & $30.96^{\circ} \mathrm{N}$ & $340.15^{\circ} \mathrm{E}$ & 1514. & 1.69 & 21.94 & $20^{-}$ & 2 & 8 \\
\hline Dec. 23,1981 & $0400: 10$ & $22.06^{\circ} \mathrm{N}$ & $008.71^{\circ} \mathrm{E}$ & 1430. & 1.32 & 04.84 & $13^{+}$ & $3-$ & 9 \\
\hline April 9, 1982 & $0212: 14$ & $38.23^{\circ} \mathrm{N}$ & $023.34^{\circ} \mathrm{E}$ & 1802. & 1.84 & 04.33 & $18^{+}$ & $3^{+}$ & 6 \\
\hline Sept. 30, 1982 & $1953: 27$ & $77.87^{\circ} \mathrm{N}$ & $041.74^{\circ} \mathrm{E}$ & 1944. & & 00.98 & $20^{-}$ & 3 & 5 \\
\hline March 10, 1983 & 1702:12 & $78.90^{\circ} \mathrm{S}$ & $087.65^{\circ} \mathrm{E}$ & 792. & & 17.31 & 13 & 2 & 4 \\
\hline
\end{tabular}




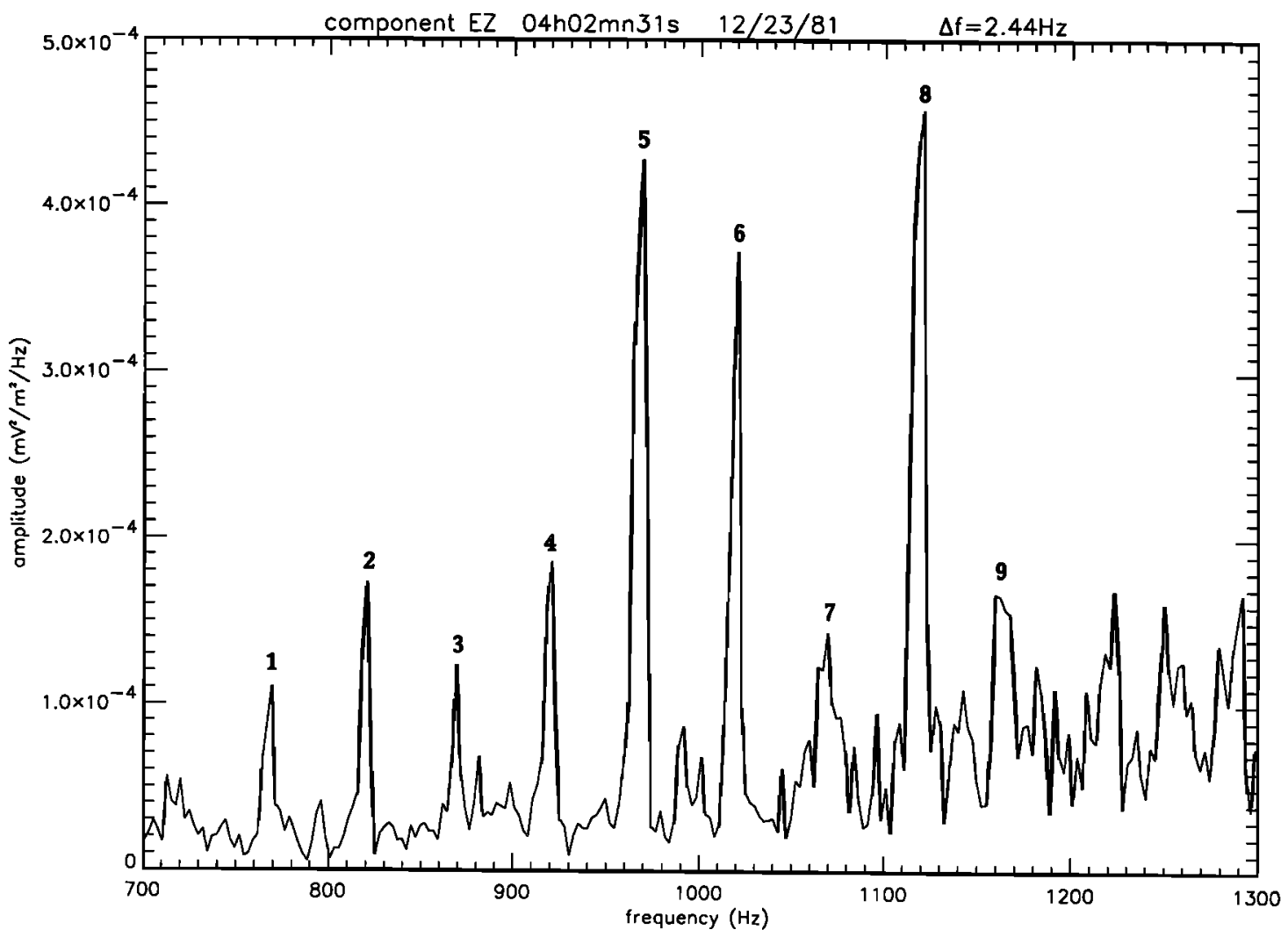

Figure 2. Frequency zoom of the spectrum of the signal of an electric component recorded on December 23, 1981 at 0402:31 UT. Peaks which are roughly distant of $50 \mathrm{~Hz}$ are numbered.

aliasing effect, Figures 3 and 4 do not show real spectra, but their symmetrics for half the sampling frequency, i.e., $2500 \mathrm{~Hz}$. This means that a peak observed at $1500 \mathrm{~Hz}$ in fact lies at $3500 \mathrm{~Hz}$. Thus, the frequencies of the lines in Figure 3 (4) are drift downwards (upwards). In all five cases, it is observed that the frequency variation has the same sense as the Earth's magnetic field.

The data corresponding to these events were recorded at three different telemetry stations: Toulouse, Tromsoe and Terre Adélie. In all cases, the interval between two adjacent lines was $50 \mathrm{~Hz}$ which is the frequency of the power system in Europe. At the longitude of Terre Adélie, Russia, China and a part of Japan are the main users of the $50 \mathrm{~Hz}$ frequency. Except for the last event on March 10,1983, all observations were recorded when the satellite was close to its apogee.

In all five cases, the spectral lines were detected on the spectrograms of the electric components. The fact that nothing was observed at the same time on the magnetic components is discussed below.

\section{Comparison With Past Observations}

The five events have several common features. The first one is that magnetospheric lines are detected during period of very low magnetic activity. Table 1 shows that $\Sigma K p$ was low during the day of the event, but low values were also observed during the previous days. Most of these days were noted as quiet by J. V. Lincoln and H.E. Coffey (Geomagnetic and solar data, various issues of Journal of Geophysical Research from 1981 to 1983). Thus magnetospheric lines are only detected when the usual natural noise is absent. This fact was already underlined for previous observations from the ground by Helliwell et al. [1975] and Matthews and Yearby [1981], as well from satellites by Bell et al. [1982]. It should also be noted that all five days corresponding to the events are weekdays when electric power consumption is larger than during weekends.

It can be seen in Table 1 that the AUREOL 3 events mainly occurred in the night sector. This does not agree with the maximum of PLHR occurrence observed by Park and Helliwell [1978], which is between 0600 and 1800 MLT. However, their ground observations were related to waves propagating in ducted mode, which are directly dependent of the power consumption that is maximum during the day. They also indicated that the frequency maximum of occurrence is around $3 \mathrm{kHz}$. The PLHR frequencies of AUREOL 3 are roughly identical if we consider that the real frequencies are equal to $5000 \mathrm{~Hz}-f$, where $f$ is the value of the frequencies observed in Figures 3 and 4.

The second common feature of the five events is that the frequency spacing of magnetospheric lines is always $50 \mathrm{~Hz}$, although they do drift. This frequency interval is expected if the lines are due to PLHR, but disagrees with previous observations. Helliwell et al. [1975], Matthews and Yearby [1981], and Bell et al. [1982] indicated that the frequency offset between two adjacent lines is close, but never exactly equal to, 50 and/or 60 Hz. A possible explanation due to the particular situation of Canada was provided by Matthews and Yearby [1981]: although the main power frequency is $60 \mathrm{~Hz}$, some plants with very high consumption use the $50-\mathrm{Hz}$ system. The two systems, 50 and 60 $\mathrm{Hz}$, which are interconnected, could generate many harmonics.

During all five events, an upwards or downwards shift in frequency has been observed. The frequency drift is different for 


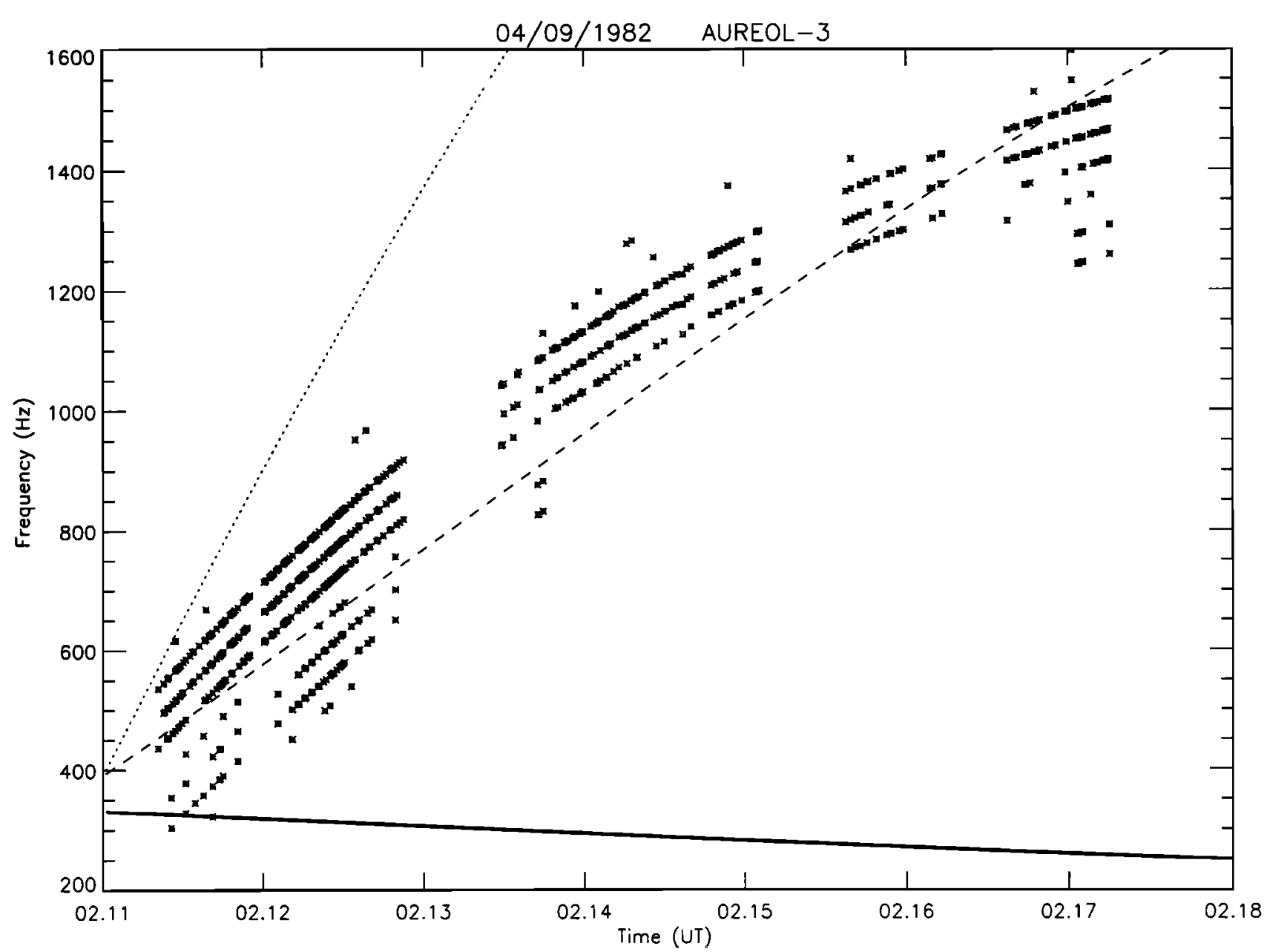

Figure 3. Extended view of the spectrogram for the event recorded on April 9, 1982 (see Figure 1). The points represent the frequencies of the peaks that were extracted from background noise. The full line represents the variation of the proton gyrofrequency given by a model. The dashed and dotted lines are related to theoretical models of waves with drifting frequencies (see text for explanation).

the five cases: it is roughly between 3 and $4 \mathrm{~Hz} / \mathrm{s}$, with a minimum of $1 \mathrm{~Hz} / \mathrm{s}$, and a maximum of $8 \mathrm{~Hz} / \mathrm{s}$. This maximum occurs during the last event of Table 1 . It is generally somewhat higher than the frequency drift previously reported: a maximum value of $50 \mathrm{~Hz} / \mathrm{min}$ was observed by Helliwell et al. [1975]; the line drifts at Halley had a maximum of $200 \mathrm{~Hz} / \mathrm{min}$, but usually were around $100 \mathrm{~Hz} / \mathrm{min}$ [Matthews and Yearby, 1981]. The satellite observations by Bell et al. [1982] showed a frequency change of approximately $22 \mathrm{~Hz} / \mathrm{min}$. Explanations of the frequency drifts of these PLHR will be discussed in the next section.

\section{Comparison With Existing Theories}

In the past, a first attempt to explain the frequency drift was to invoke the Doppler shift due to the satellite velocity. If we consider that the wave normal vector $\mathbf{k}$ is nearly parallel to the satellite velocity vector $v_{\mathbf{s}}$, the maximum Doppler frequency shift $\Delta f$ is given by

$$
\Delta f=\frac{k V_{s}}{2 \pi}=\frac{n f V_{s}}{r}
$$

where $c$ is the velocity of light, and $n$ is the refractive index. The maximum value of $n$ in the whistler mode approximation is obtained when $k$ is nearly perpendicular to the Earth's magnetic field. Thus $n$ is given by

$$
n=\left(\frac{R L}{S}\right)^{1 / 2}
$$

where $R, L$, and $S$ are the plasma coefficients defined by Stix [1962]. As the events were recorded when the satellite was at relatively high altitude, only protons were considered to evaluate equation (4). Equation (3) has been calculated for the December 23,1981 , event. The proton gyrofrequency $f_{k J}$ varied as in Figure 4 , the velocity of the satellite $v_{s}$ was $6.8 \mathrm{~km} / \mathrm{s}$, and the plasma frequency $f_{p}$ was estimated to be $900 \mathrm{kHz}$. It was found that, at 0358:00 UT, the Doppler shift $\Delta f=6.3 \mathrm{~Hz}$ for $f=3500 \mathrm{~Hz}$, and at 0402:40 UT for the same frequency, $\Delta f=5.6 \mathrm{~Hz}$. Considering that the PLHR were observed in a frequency band of $500 \mathrm{~Hz}, \Delta f$ was estimated at a given time for two different frequencies. It was found that at 0358:00 UT, $\Delta f=6.7 \mathrm{~Hz}$ for $f=3700 \mathrm{~Hz}$, and $\Delta f=5.7 \mathrm{~Hz}$ for $f=3200 \mathrm{~Hz}$. At $0402: 40$ UT, the values are $\Delta f=6.1 \mathrm{~Hz}$ for $f=3800 \mathrm{~Hz}$, and $\Delta f=7.1$ $\mathrm{Hz}$ for $f=4300 \mathrm{~Hz}$. Therefore two conclusions are clear: first, the Doppler shift does not explain the frequency drift observed during the events as the difference of shift between 0358:00 UT and 0402:40 UT is only $0.7 \mathrm{~Hz}$ at a given frequency; second, the 


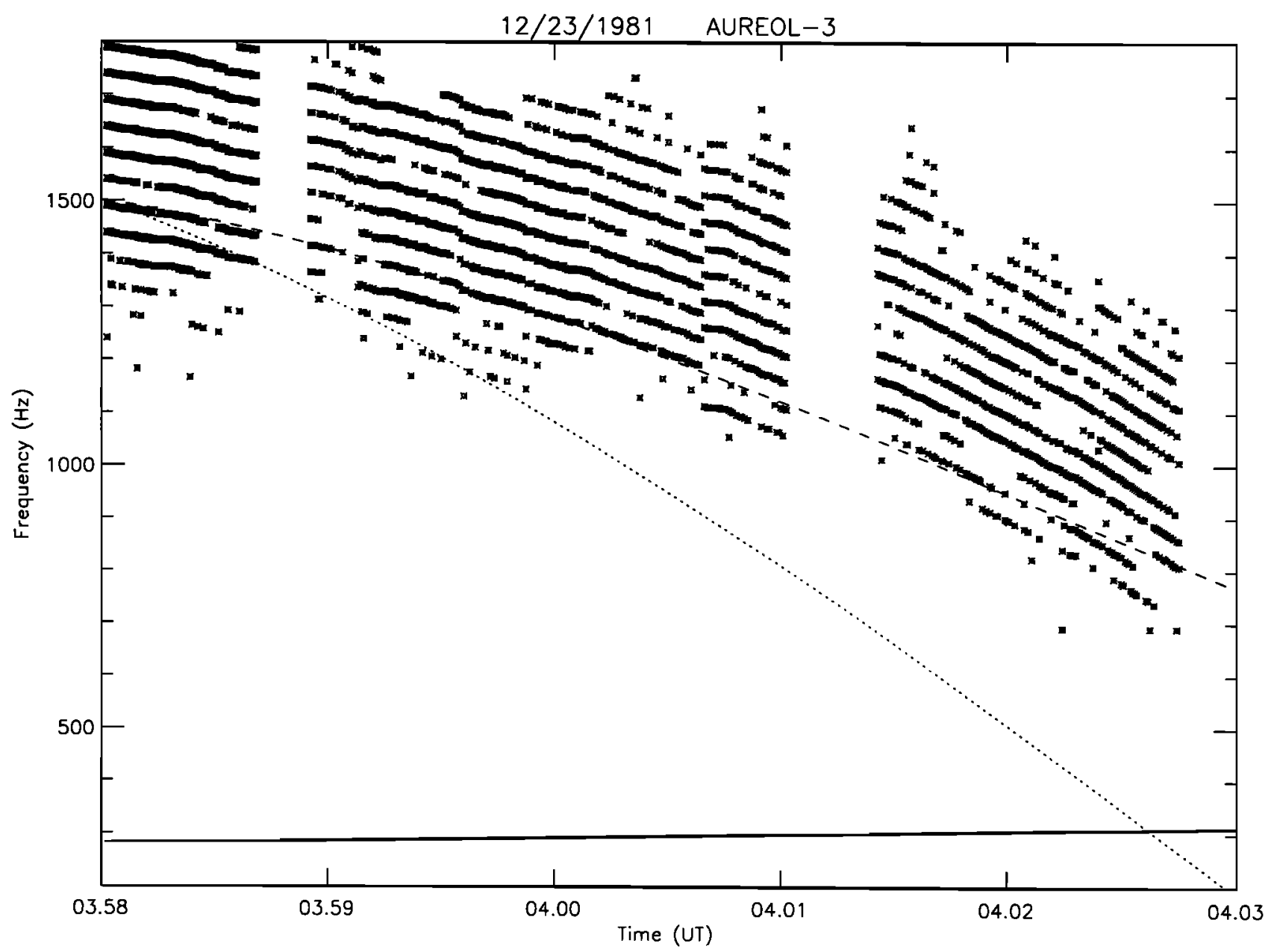

Figure 4. Same as in Figure 3, but for the event recorded on December 23, 1981.

Doppler shift does not destroy the $50-\mathrm{Hz}$ interval between the PLHR, as at any moment, the maximum difference between the $\Delta f$ values of lines separated by $500 \mathrm{~Hz}$ is $1 \mathrm{~Hz}$.

To study the triggering of discrete VLF emissions, Helliwell [1967] considered the wave-particle interaction involving cyclotron resonance. In an inhomogeneous magnetic field, the electrons cannot stay in resonance with waves, and he calculated the variation of the wave frequency $\mathrm{d} f$ which is necessary to maintain an interaction:

$$
d f=\frac{3 \lambda}{1+2 \lambda}\left[1+\frac{(1-\lambda)}{3} \tan ^{2} \alpha\right] d f_{c}
$$

where $\lambda=f / f_{c}, f_{c}$ is the electron gyrofrequency, and $\alpha$ is the pitch angle of the electrons. In this equation, it is supposed that the wave normal vector $\mathbf{k}$ is in the direction of the Earth's magnetic field. It means that equation (5) was obtained with $n^{2}=R$. In his calculation, Helliwell [1967] considered an interaction taking place in equatorial regions; but it might take place elsewhere. The importance of off-equatorial resonance interactions of coherent whistler mode waves and electrons was underlined by Neubert et al. [1987], who showed that pitch angle scattering due to cyclotron resonance is enhanced in the topside ionosphere where these PLHR events are located. Although other explanations cannot be ruled out [Bell et al., 1982], it is hypothesized that the observed PLHR is amplified in the topside ionosphere rather than at the magnetic equatorial plane. This interpretation is supported by the following two points: first, the electrostatic nature of the waves suggests that the stimulation does not take place very far from the satellite; second, the frequency drift rate shows a relation with the local magnetic field, as it will be examined in the next paragraph.

The theoretical equation (5) has been used, with the experimental conditions of the April 9 event, to calculate the frequency drift of one line. The initial frequency at 0211 UT was $4600 \mathrm{~Hz}$. The time step used to resolve the equation was $1 \mathrm{~s}$. The curve that was obtained is plotted as a dotted line on Figure 3 and does not represent the variation of the frequency $f$, but the variation of the frequency $5000 \mathrm{~Hz}-f$. The value of $\alpha$ was chosen to obtain the best fit with the PLHR. It corresponds to a pitch angle nearly equal to 0 . The same process was used for the December 23 event with an initial frequency at 0358 UT equal to $3500 \mathrm{~Hz}$. The result is plotted as a dotted curve in Figure 4. In both cases, we observe that, as time goes on, the theoretical curves rapidly become different from the observed ones. Thus more plasma parameters have been considered in the simulation. Following the work of Helliwell [1967], a new term was introduced by Sudan and Ott [1971] to account for variation of electronic density $n_{0}$ along the wave path. The variation of frequency $\mathrm{d} f$ ' is now given by 


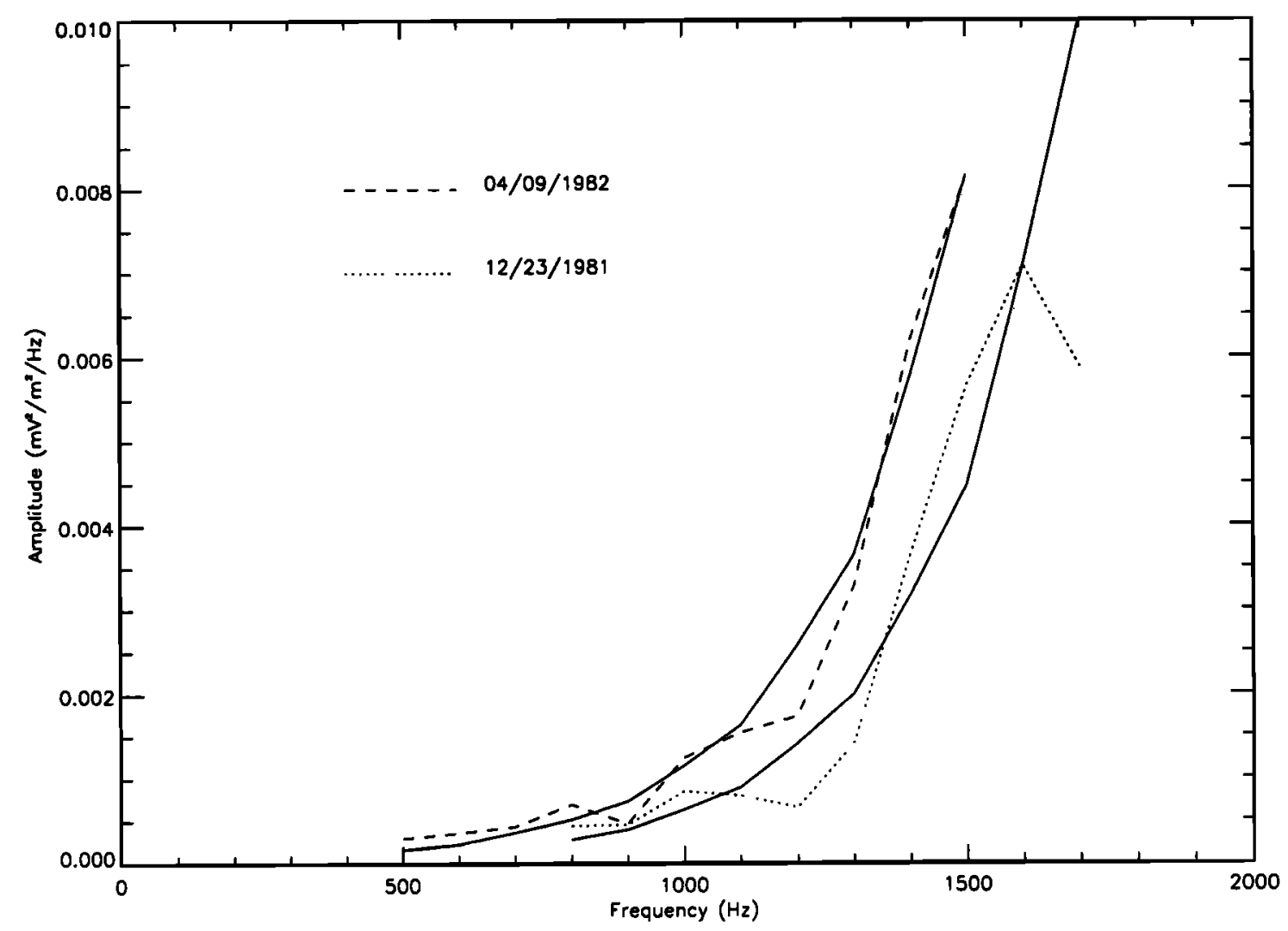

Figure 5. The dashed and dotted curves represent the average spectrum of the events recorded on April 9, 1982, and on December 23, 1981, respectively. These spectra have been averaged for frequency and time. The solid curves are related to the slope of the antialiasing filter (see text for explanation).

$$
d f^{\prime}=d f-\frac{2 \lambda(1-\lambda)}{1+2 \lambda} f_{c} \frac{d n_{0}}{n_{0}}
$$

where $\mathrm{d} f$ is calculated with equation (5). Using the same experimental conditions as before, the frequency shifts have been calculated for the two events, and the variation of density was adjusted to fit the observations. The curves are shown as dashed lines in Figures 3 and 4 . Good agreement is obtained between experimental and theoretical curves, especially for the December 23 event. The variation of electronic density, which is needed to attain this result, is relatively small: $0.3 \%$ during the $7 \mathrm{~min}$ of the April 9 event, and $0.07 \%$ during the $5 \mathrm{~min}$ of the December 23 event.

A second correction to the variation of the wave frequency $\mathrm{d} f$ given by equation (5) was introduced by Matsumoto [1979]:

$$
d f^{\prime \prime}=d f+\Delta u d l
$$

The value of $\Delta u$, which represents a non linear effect resulting from particle trapping in phase potential wells, is limited by

$$
\frac{-3 \lambda}{2 \pi(2 \lambda+1)} \omega_{i}^{2} \leq \Delta u \leq \frac{3 \lambda}{2 \pi(2 \lambda+1)} \omega_{t}^{2}
$$

Here, $\omega_{\imath}$ is a wave trapping frequency defined by

$$
\omega_{1}^{2}=k v_{\perp} e B_{w} / m
$$

where $v_{\perp}$ is the perpendicular velocity of the resonant particle, $e$ and $m$ the electronic charge and mass, respectively, and $B_{w}$ the wave magnetic field. The inequality of equation (8) expresses the necessary condition to have resonant electrons phase-trapped in waves [Matsunoto, 1979]: it is due to the fact that the secondorder resonance angle introduced by Nunn [1971] can take an arbitrary value between $-\pi / 2$ and $\pi / 2$ (in Helliwell's treatment, this angle is zero). Thus it is always possible to find a value of $\Delta u$ in equation (7), that will give a theoretical curve similar to that obtained with equation (6). Concerning the December 23 case, it is found that $\Delta u=-1.6 \mathrm{~Hz} / \mathrm{s}$. However, it must be noted that the possible maximum value of $\Delta u$ is much too large when we consider the intensity of the wave magnetic field $B_{w}$ recorded during the events. With the experimental conditions of the December 23 case at $0358 \mathrm{UT}$, and considering the gyroresonance relation $k v_{1}=2 \pi\left(f_{c}-f\right)$ to calculate $\omega_{1}$ in the equation (9), the maximum value of $\Delta u$ is $49 \mathrm{~Hz} / \mathrm{s}$ if we suppose that the pitch angle $\alpha=45^{\circ}\left(\tan \alpha=v_{\perp} / v_{1}\right)$. It is 6 times larger than the maximum drift that is observed. This rough estimate of the maximum $\Delta u$ is obtained with the experimental value $B_{w}=0.22 \mathrm{pT}$, but no significant peak is observed in the magnetic spectrum around $1500 \mathrm{~Hz}$.

Another possibility to explain the frequency drift was given by Matthews et al. [1984a, 1984b], who used a numerical simulation model to study the equatorial particle interaction with a whistler mode wave in the inhomogeneous geomagnetic field. They showed that a wave which passes several times in the equatorial amplification region has a maximum growth at a frequency that 
shifts upwards. The frequency shift is estimated to be $\approx f_{1} / 2$, where $f_{t}=\omega_{1} / 2 \pi$ and $\omega_{t}$ is given by equation (9). With the parameter values of the December 23 event presented above, $\omega_{1}$ $\approx 20 \mathrm{~Hz}$. In their model the amplified wave at the frequency $f+$ $f_{1} / 2$ propagates from the equator, is reflected in the ionosphere (where it could be observed by a low-altitude satellite), and returns to the equator to undergo another shift. Considering that there is a frequency shift of $\approx f_{t} / 2$ per hop, and that one hop travel time is $3 \mathrm{~s}$, it gives a frequency drift of $\approx 3 \mathrm{~Hz} / \mathrm{s}$ which is rather consistent with the observations.

A similar result was obtained by Nunn [1986]: using a different approach, he studied the nonlinear sideband stability of different ducted whistler waves in cyclotron resonance with electrons. In the PLHR case, he considered that the interaction takes place around the magnetospheric equator at $L=4$ and found a maximum shift $\Delta f=2.4 \mathrm{~Hz}$ when $B_{w}=2.7 \mathrm{pT}$ and $f=2858$ Hz. With these experimental conditions (which are not exactly relevant for the AUREOL 3 observations), a maximum upward drift rate of $30 \mathrm{~Hz} / \mathrm{min}$ is expected, considering that the PLHR frequency increases by $\Delta f$ on each equatorial pass. But, the AUREOL 3 events that were observed at high latitudes do not support the fact that this interaction occurs near the equator. Moreover, Nunn [1986] noted that this mechanism needed sufficient amplitudes of the wave magnetic field $B_{w}(>0.5 \mathrm{pT})$, which is not the case of our events.

Two reasons may explain that the PLHR are not observed in the magnetic spectrograms. First, the values of the wave magnetic field $\left(1.510^{-4} \gamma / \mathrm{Hz}^{1 / 2}\right.$ at $\left.1000 \mathrm{~Hz}\right)$ are about the same as the noise level of the experiment at the frequencies of observation. Second, the PLHR have certainly been subjected to other mechanisms that would have increased their electrostatic part. This could be related to the general problem of sideband generation; sidebands of electrostatic nature are mainly observed around the pulses of ground-based VLF transmitters. The possible generation mechanisms are (see, for example, Bell [1985], Bell and Ngo [1988] and references therein): interhemispheric propagation, scattering from ionospheric irregularities, nonlinear scattering involving a parametric instability, and whistler mode sideband instability. Interhemisphere propagation is not applicable to our events, and the scattering from ionospheric irregularities generally induces symmetric sidebands around the carrier. No other frequency component in the spectra can be considered to produce a parametric interaction with the input waves, but the fact that many spectral lines maintain a constant frequency gap during a certain time, may be the result of a parametric interaction between them. On the other hand, the whistler mode sideband instability [Nunn, 1971; Brinca, 1972a, 1972b; Denavit and Sudan, 1975] is linked to the same nonlinear interaction process between coherent waves and particles that we saw before. The theory also predicts that the lower sideband is damped. Thus the most likely mechanism that is proposed to explain the AUREOL 3 events, assumes a nonlinear interaction with frequency drift, as was been shown with the equations (5)-(7); this whistler mode instability simultaneously induces the intense electrostatic part of the sidebands that are observed close to the region of generation.

\section{Conclusions}

Several observations of PLHR were made with the low-altitude AUREOL 3 satellite, whose common features are that the lines drift together and have a roughly constant frequency spacing of $50 \mathrm{~Hz}$, their frequency range is mainly between 3 and $4 \mathrm{kHz}$, they are only observed with the electric components, and they are observed during periods of very quiet magnetic activity.
The frequency drift of these PLHR is not due to a Doppler effect but can be explained by considering a whistler mode instability during a gyroresonance interaction between such coherent waves and particles in an inhomogeneous medium. The mechanism is supposed to take place in the topside ionosphere. Due to their intensity, only triggered electrostatic sidebands are observed.

Such observations are generally rare (only five cases were detected among 940 different telemetry records from the AUREOL 3 satellite). This is not very surprising because, due to wave experiment constraints, they occurred in a frequency band that is not often correctly surveyed by magnetospheric satellites, and because the interaction mechanism that enhances these emissions mainly occurs when magnetic activity is very low. Moreover, PLHR will be masked when intense natural noise is present in spectrograms. But, even if they are rarely observed, PLHR are omnipresent in the magnetosphere and play a role in wave-particle interaction.

Acknowledgments. The AUREOL 3 program was undertaken jointly by the Centre National d'Etudes Spatiales (CNES) in France, the Space Research Institute (IKI) and INTERCOSMOS in Russia. The author thanks J. J. Berthelier, principal investigator of the VLF experiment, for use of the data. He also expresses his thanks to D. Lagoutte, G. Pepin and J. L. Rauch, who helped in data handling.

The editor thanks K.-I. Nishikawa and another referee for their assistance in evaluating this paper.

\section{References}

Bell, T.F., High-amplitude VLF transmitter signals and associated sidebands observed near the magnetic equatorial plane on the ISEE 1 satellite, J. Geophys. Res., 90, 2792$2806,1985$.

Bell, T.F., and H.D. Ngo, Electrostatic waves stimulated by coherent VLF signals propagating in and near the inner radiation belt, J. Geophys. Res., 93, 2599-2618, 1988.

Bell, T.F., and H.D. Ngo, Electrostatic lower hybrid waves excited by electromagnetic whistler mode waves scattering from planar magnetic-field-aligned plasma density irregularities, J. Geophys. Res., 95, 149-172, 1990.

Bell, T.F., J.P. Luette, and U.S. Inan, ISEE 1 observations of VLF line radiation in the Earth's magnetosphere, J. Geophys. Res., 87, 3530-3536, 1982.

Berthelier, J.J., et al., Measurements of the VLF electric and magnetic components of waves and DC electric field on board the AUREOL 3 spacecraft: The TBF-ONCH experiment, $A n n$. Geophys., 38, 643-667, 1982.

Brinca, A.L., Whistler side-band growth due to nonlinear waveparticle interaction, J. Geophys. Res., 77, 3508-3523, 1972a.

Brinca, A.L., Sideband growth in nonlinear Landau wave-particle interaction, J. Plasina Phys., 7, 385-402, 1972b.

Bullough, K., A.R.L. Tatnall, and M. Denby, Man-made ELF/VLF emission and the radiation belts, Nature, 260, 401$403,1976$.

Bullough, K., T.R. Kaiser, and H.J. Strangeways, Unintentional man-made modification effects in the magnetosphere, $J$. Atmos. Terr. Phys., 47, 1211-1223, 1985.

Carlson, C.R., R.A. Helliwell, and U.S. Inan, Space-time evolution of whistler mode wave growth in the magnetosphere, J. Geophys. Res., 95, 15,073-15,089, 1990.

Denavit, J., and R.N. Sudan, Whistler sideband instability, Phys. Fluids, 18, 575-584, 1975.

Helliwell, R.A., A theory of discrete VLF emissions from the magnetosphere, J. Geophys. Res., 72, 4773-4790, 1967.

Helliwell, R.A., VLF wave stimulation experiments in the magnetosphere from Siple station, Antarctica, Rev. Geophys., $26,551-578,1988$. 
Helliwell, R.A., J.P. Katsufrakis, T.F. Bell and R. Raghuram, VLF line radiation in the Earth's magnetosphere and its association with power system radiation, J. Geophys. Res., 80, 4249-4258, 1975.

Kikuchi, H., Overview of power-line radiation and its coupling to the ionosphere and magnetosphere, Space Sci. Rev., 35, 33$41,1983$.

Kimura, I., H. Higuchi, K. Hashimoto, N. Sato, and T. Matsudo, Relationship between power line radiation and VLF wave activities observed in Iceland, Mem. Nall. Inst. Polar Res. Spec. Issue, Jpn., 47, 97-108, 1987.

Koons, H.C., M.H. Dazey, and B.C. Edgar, Satellite observation of discrete VLF line radiation within transmitter-induced amplification bands, J. Geophys. Res., 83, 3887-3889, 1978.

Matsumoto, H., Nonlinear whistler mode interactions and triggered emissions in the magnetosphere: A review, in Wave Instabilities in Space Plasma, pp. 163-190, D. Reidel, Norwell, Mass., 1979.

Matthews, J.P., and K. Yearby, Magnetospheric VLF line radiation observed at Halley, Antarctica, Planet. Space Sci., 29, 97-106, 1981

Matthews, J.P., Y. Omura, and H. Matsumoto, Some PLHR phenomena and their explanation, in International Wroclaw Sympositun on Electromagnetic Compatibility, pp. 55-59, Technical University of Wroclaw, Poland, 1984a.

Matthews, J.P., Y. Omura, and H. Matsumoto, A study of particle trapping by whistler mode waves in the geomagnetic field: The early development of the VLF quiet band, $J$. Geophys. Res., 89, 2275-2287, 1984b.

Molchanov, O.A., M. Parrot, M.M. Mogilevsky, and F. Lefeuvre, A theory of PLHR emissions to explain the weekly variations of ELF data observed by a low-altitude satellite, Ann. Geophys., 9, 669-680, 1991.

Neubert, T., T.F. Bell, and L.R.O. Storey, Resonance between coherent whistler mode waves and electrons in the topside ionosphere, J. Geophys. Res., 92, 255-265, 1987.

Nunn, D., A theory of VLF emissions, Planet. Space Sci., 19, 1141-1167, 1971.

Nunn, D., The sideband instability of electrostatic waves in an inhomogeneous medium, Planet. Space Sci., 21, 67-88, 1973.

Nunn, D., The quasistatic theory of triggered VLF emissions, Planet. Space Sci., 32, 325-350, 1984.

Nunn, D., A nonlinear theory of sideband stability in ducted whistler mode waves, Planet. Space Sci., 34, 429-451, 1986.

Omura, Y., D. Nunn, H. Matsumoto, and M.J. Rycroft, A review of observational, theoretical and numerical studies of VLF triggered emissions, J. Atmos. Terr. Phys., 53, 351-368, 1991.
Park, C.G., and D.C.D. Chang, Transmitter simulation of power line radiation effects in the magnetosphere, Geophys. Res. Lett., 5, 861-864, 1978.

Park, C.G., and R.A. Helliwell, Magnetospheric effects of power line radiation, Science, 200, 727-730, 1978.

Park, C.G., and R.A. Helliwell, Power line radiation in the magnetosphere, Adv. Space Res., 1, 423-437, 1981.

Parrot, M., World map of ELF/VLF emissions as observed by a low-orbiting satellite, Ann. Geophys., 8, 135-146, 1990.

Parrot, M., O.A. Molchanov, M.M. Mogilevski, and F. Lefeuvre, Daily variations of ELF data observed by a low altitude satellite, Geophys. Res. Lett., 18, 1039-1042, 1991.

Sà, L.A.D., A wave-particle-wave interaction mechanism as a cause of VLF triggered emissions, J. Geophys. Res., 95, $12,277-12,286,1990$.

Stix, T. H., The Theory of Plasma Waves, pp. 9-13, McGrawHill, New York, 1962.

Sudan, R.N., and E. Ott, Theory of triggered VLF emissions, J. Geophys. Res., 76, 4463-4476, 1971.

Tanaka, Y., D. Lagoutte, M. Hayakawa, F. Lefeuvre, and S. Tajima, Spectral broadening of VLF transmitter signals and sideband structure observed on Aureol 3 satellite at middle latitudes, J. Geophys. Res., 92, 7551-7559, 1987.

Tatnall, A.R.L., J.P. Matthews, K. Bullough, and T.R. Kaiser, Power-line harmonic radiation and the electron slot, Space Sci. Rev., 35, 139-173, 1983.

Tomizawa, I., and T. Yoshino, Power line radiation over northern Europe observed on the balloon $\mathrm{B}_{15}-1 \mathrm{~N}, \mathrm{Mem}$. Natl. Inst. Polar Res. Spec. Issue, Jpn., 31, 115-123, 1984.

Tomizawa, I., and T. Yoshino, Power line radiation observed by the satellite OHZORA, J. Geomagn. Geoelectr., 37, 309-327, 1985.

Yearby, K.H., A.J. Smith, T.R. Kaiser, and K. Bullough, Power line harmonic radiation in Newloundland, J. Almos. Terr. Phys., 45, 409-419, 1983.

M. Parrot, Laboratoire de Physique et Chimie de l'Environnement, CNRS, 3A, Avenue de la Recherche Scientifique, 45071 Orléans cedex 02, France.

(Received June 11, 1993; revised August 16, 1993; accepted August 31, 1993.) 